\title{
OBTENÇÃO E CARACTERIZAÇÃO DE NANOFIBRAS CERÂMICAS DE ÓXIDO DE ZINCO POR ELETROFIAÇÃO
}

\author{
M. C. da ROCHA ${ }^{1}$, R. C. NONATO ${ }^{1}$ e A. R. MORALES ${ }^{1}$
}

${ }^{1}$ Universidade Estadual de Campinas, Faculdade de Engenharia Química

E-mail para contato: morales@feq.unicamp.br,m147342@dac.unicamp.br

\begin{abstract}
RESUMO - Nos dias atuais há um grande interesse em nanomateriais, dentre eles as nanofibras, pois elas apresentam diversas propriedades atrativas, como elevadas propriedades térmicas e mecânicas, além de alta resistência à corrosão. Neste trabalho, pretende-se estudar as condições de processo para a obtenção de nanofibras de óxido de zinco $(\mathrm{ZnO})$ por eletrofiação (electrospinning), bem como caracterizá-las por Microscopia Eletrônica de Varredura e Difração de Raios X, com posterior discussão de resultados focando sua futura aplicação na produção de nanocompósitos de matriz polimérica. As nanofibras de $\mathrm{ZnO}$ foram escolhidas graças a sua versatilidade de aplicações.
\end{abstract}

\section{INTRODUÇÃO}

As fibras cerâmicas apresentam propriedades atrativas para a indústria, uma vez que são inertes, apresentam alta rigidez, além de serem amplamente conhecidas por suas excelentes propriedades mecânicas e térmicas, bem como sua resistência à corrosão. Tais características as tornam amplamente utilizadas em diversos campos, destacando-se como um dos principais reforços utilizados em compósitos, cuja confecção ganhou um destaque muito importante nos últimos anos. Dentre as aplicações mais conhecidas, ressalta-se a fabricação de componentes eletrônicos, componentes de distribuição de medicamentos, aplicações ambientais, em processos de filtração e separação, como substrato para catálise, sensores e eletrodos para conversão de energia ou dispositivos de armazenamento (Ramaseshan et al., 2007).

Com o passar dos anos, muitos métodos foram desenvolvidos para se produzir materiais cerâmicos na forma de nanoestruturas, uma vez que materiais cerâmicos nanoestruturados são ainda mais atrativos devido a sua maior área interfacial. Uma técnica simples e versátil é denominada eletrofiação, conhecida do inglês como electrospinning, em que é possível fabricar-se estruturas unidimensionais (1D), como nanofibras, em uma escala industrial e com diâmetro, composição e morfologia controláveis. Grandes comprimentos, grande área interfacial e estruturas porosas hierárquicas são algumas das características e propriedades apresentadas nas fibras cerâmicas produzidas por meio da eletrofiação (Li; Xia, 2003). Ressalta-se, inclusive, que as mesmas podem ser preparadas como membranas por meio deste método, tornando conveniente sua manipulação durante suas aplicações.

Dentre as nanofibras fabricadas por eletrofiação destacam-se as nanofibras de Óxido de Zinco $(\mathrm{ZnO})$, devido às suas boas propriedades mecânicas, além de sua grande resistência térmica e à corrosão. Muito se estuda o $\mathrm{ZnO}$ na forma de pó e não na forma de nanofibras (Sim et al., 2005; Tjong; Liang 2006). Seu uso vai desde aplicações para sensores de gases até 
como pomada devido às suas propriedades antissépticas (Moorer et al., 1982; Wei et al., 2011), sendo o uso na área médica muito explorado, principalmente por conta de seu poder antibactericida (Lee, 2009).

\section{JUSTIFICATIVA E OBJETIVOS}

Atualmente, devido às propriedades e características atrativas, tem-se um grande interesse no desenvolvimento de novas aplicações para as nanofibras cerâmicas, especialmente aquelas produzidas por meio de eletrofiação, entretanto, a produção das mesmas é pouco explorada no Brasil, e não são encontrados relatos de estudos de nanofibras de $\mathrm{ZnO}$ por instituições nacionais.

Dessa maneira, o domínio de métodos eficazes para a fabricação de nanofibras cerâmicas é tido como de estratégica importância, visto que as mesmas podem ser utilizadas em diversos campos, muitos deles ainda em desenvolvimento e com promissoras aplicações futuras. As nanofibras de $\mathrm{ZnO}$ foram escolhidas por suas aplicações diversas já estudadas, além de sua possibilidade de uso em outros campos.

Desta forma, tem-se como objetivo do trabalho o estudo das condições de processo para a fabricação de nanofibras cerâmicas de $\mathrm{ZnO}$ por meio de eletrofiação, baseado na realização de um planejamento fatorial para determinar a condição de processo ótima no meio mais favorável, visando a um maior rendimento e obtenção de fibras com diâmetros menores e mais homogêneos. Tem-se também como objetivo a caracterização inicial por MEV e Difração de Raios X das nanofibras de $\mathrm{ZnO}$ obtidas. Este estudo é realizado como parte do trabalho de doutorado de um aluno do grupo de pesquisa.

\section{METODOLOGIA}

\subsection{Preparação da solução}

As nanofibras cerâmicas de $\mathrm{ZnO}$ foram fabricadas por meio da eletrofiação. Nesta técnica, partiu-se de uma solução onde foram diluídos os precursores da fibra cerâmica, no caso do $\mathrm{ZnO}$ utilizou-se o Acetato de Zinco Diidratado, o polímero Acetato de Polivinila (PVA) e como solvente a água. Essa solução foi colocada numa seringa fixada em uma bomba diante do aparelho. A partir de uma diferença de potencial, ocorre a deposição desses elementos da seringa em uma placa de cobre revestida com uma folha de alumínio. Esse material foi então calcinado, restando somente a nanofibra cerâmica.

O método de preparação da solução consistiu na solubilização dos precursores em água destilada. A concentração em massa utilizada foi de 7,5\% de PVA e 7,5\% de AcZn. Houve a agitação à temperatura ambiente durante 6 horas para garantir-se a homogeneidade adequada. A solução permaneceu em repouso por um período de 24 horas.

\subsection{Eletrofiação}

As características morfológicas das fibras cerâmicas obtidas pela eletrofiação, como dimensão, formato e diâmetro, são fortemente influenciadas pelos parâmetros envolvidos no processo de obtenção. Dessa forma, a eletrofiação caracteriza-se como uma técnica complexa, 
entretanto possui um princípio de funcionamento simples (Gensheimer et al.; 2007). Ela consiste na preparação da solução polimérica constituída dos precursores da fibra cerâmica, que é posteriormente introduzida em uma seringa. Esta é ligada à uma bomba que permite a solução ser expelida com uma vazão constante. Dois eletrodos conectados a uma fonte de alta voltagem são utilizados para gerar uma diferença de potencial entre a agulha e um coletor de metal revestido por uma folha de alumínio localizado à frente da seringa. Um dos eletrodos é conectado na ponta da agulha e o outro fixado no coletor de metal (Li et al.; 2006), de forma que a solução seja atraída pelo coletor devido à diferença de potencial gerada. Durante essa etapa, é verificada a evaporação do solvente, que permite a deposição da fibra seca e normalmente na forma de manta sobre a folha de alumínio que reveste o coletor metálico (Wannatong et al., 2004).

Logo, o aparato experimental necessário para a eletrofiação inclui uma seringa dotada de uma agulha; uma fonte de alta voltagem; uma bomba de vazão; um coletor de metal (Ramakrishna et al., 2005) e uma folha de alumínio para revestimento do coletor. Uma fita isolante também pode ser necessária, se porventura um isolamento for necessário.

Almeja-se que as nanofibras produzidas por eletrofiação sejam contínuas, detenham diâmetros com valores estáveis e controláveis, bem como apresentem uma superfície livre de defeitos ou com defeitos controláveis (Nista, 2012). Tais características são afetadas por diversos parâmetros que influenciam o processo de eletrofiação, podendo-se citar: as propriedades da solução (viscosidade, condutividade elétrica e tensão de superfície) que influenciam principalmente o diâmetro e morfologia das fibras, as condições de processo (voltagem, distância do coletor, vazão da solução e efeito do coletor) e os parâmetros ambientais (umidade e pressão).

No presente trabalho estudou-se os seguintes parâmetros de processo relativos à eletrofiação: voltagem, distância do coletor e vazão da solução. Além do tempo de calcinação e da temperatura de calcinação que serão os responsáveis pela formação do $\mathrm{ZnO}$.

\subsection{Caracterização das nanofibras}

Microscopia Eletrônica de varredura: As nanofibras foram observadas por Microscopia Eletrônica de Varredura, com o intuito de se observar imagens de alta resolução, com uma aparência tridimensional, da superfície da amostra para enfim se ter uma melhor ideia de sua estrutura final. A morfologia das fibras foi observada antes e após a calcinação, podendo-se confirmar, assim, a formação de fibras e se obter informações sobre seu diâmetro. Posteriormente, com o uso do software ImageJ 1.47, serão obtidas 50 medições de diâmetro para a determinação do diâmetro médio das fibras de cada amostra.

Difração de Raios X: A difração de raios X será realizada antes e após a calcinação com o intuito de confirmar se houve a formação de $\mathrm{ZnO}$ após a calcinação. Dessa forma, tem-se como objetivos a caracterização dos reagentes utilizados e posterior identificação da conversão do AcZn em $\mathrm{ZnO}$.

\subsection{Proposta de trabalho}


Estudos preliminares do grupo de pesquisa indicaram que a confecção de nanofibras de $\mathrm{ZnO}$ foi otimizada com concentrações de 7,5 \% de PVA e 7,5 \% de AcZn. Com essas informações, fez-se um planejamento fatorial $2^{5}$ visando otimizar os parâmetros de processo, além de maximizar a quantidade de fibra formada. As variáveis em questão bem como seus limites superior e inferior encontram-se na Tabela 1.

Tabela 1 - Planejamento fatorial $2^{5}$

\begin{tabular}{|c|c|c|c|c|c|c|c|c|c|c|}
\hline & \multicolumn{5}{|c|}{ Variáveis codificadas } & \multicolumn{4}{|c|}{ Variáveis decodificadas } & \multirow[b]{2}{*}{$\begin{array}{c}\text { Temperatura } \\
\text { calcinação } \\
\left({ }^{\circ} \mathrm{C}\right)\end{array}$} \\
\hline & $\begin{array}{c}\text { Distância } \\
\text { coletor }(\mathrm{cm})\end{array}$ & $\begin{array}{c}\text { Vazão } \\
\text { (mL/min) }\end{array}$ & $\begin{array}{c}\text { Voltagem } \\
(\mathrm{kV})\end{array}$ & $\begin{array}{c}\text { Tempo } \\
\text { calcinação (h) }\end{array}$ & $\begin{array}{l}\text { Temperatura } \\
\text { calcinação }\left({ }^{\circ} \mathrm{C}\right)\end{array}$ & $\begin{array}{c}\text { Distância } \\
\text { coletor }(\mathrm{cm})\end{array}$ & $\begin{array}{c}\text { Vazão } \\
\text { (mL/min) }\end{array}$ & Voltagem (kV) & $\begin{array}{c}\text { Tempo } \\
\text { calcinação } \\
\text { (h) }\end{array}$ & \\
\hline A1 & 1 & 1 & 1 & 1 & 1 & 15 & 0,0388 & 22 & 6 & 500 \\
\hline $\mathrm{A} 2$ & 1 & 1 & 1 & 1 & -1 & 15 & 0,0388 & 22 & 6 & 300 \\
\hline A3 & 1 & 1 & 1 & -1 & 1 & 15 & 0,0388 & 22 & 3 & 500 \\
\hline A4 & 1 & 1 & 1 & -1 & -1 & 15 & 0,0388 & 22 & 3 & 300 \\
\hline B1 & 1 & 1 & -1 & 1 & 1 & 15 & 0,0388 & 17 & 6 & 500 \\
\hline $\mathrm{B} 2$ & 1 & 1 & -1 & 1 & -1 & 15 & 0,0388 & 17 & 6 & 300 \\
\hline B3 & 1 & 1 & -1 & -1 & 1 & 15 & 0,0388 & 17 & 3 & 500 \\
\hline B4 & 1 & 1 & -1 & -1 & -1 & 15 & 0,0388 & 17 & 3 & 300 \\
\hline $\mathrm{C} 1$ & 1 & -1 & 1 & 1 & 1 & 15 & 0,0194 & 22 & 6 & 500 \\
\hline $\mathrm{C} 2$ & 1 & -1 & 1 & 1 & -1 & 15 & 0,0194 & 22 & 6 & 300 \\
\hline $\mathrm{C} 3$ & 1 & -1 & 1 & -1 & 1 & 15 & 0,0194 & 22 & 3 & 500 \\
\hline C4 & 1 & -1 & 1 & -1 & -1 & 15 & 0,0194 & 22 & 3 & 300 \\
\hline D1 & 1 & -1 & -1 & 1 & 1 & 15 & 0,0194 & 17 & 6 & 500 \\
\hline D2 & 1 & -1 & -1 & 1 & -1 & 15 & 0,0194 & 17 & 6 & 300 \\
\hline D3 & 1 & -1 & -1 & -1 & 1 & 15 & 0,0194 & 17 & 3 & 500 \\
\hline D4 & 1 & -1 & -1 & -1 & -1 & 15 & 0,0194 & 17 & 3 & 300 \\
\hline E1 & -1 & 1 & 1 & 1 & 1 & 10 & 0,0388 & 22 & 6 & 500 \\
\hline E2 & -1 & 1 & 1 & 1 & -1 & 10 & 0,0388 & 22 & 6 & 300 \\
\hline E3 & -1 & 1 & 1 & -1 & 1 & 10 & 0,0388 & 22 & 3 & 500 \\
\hline E4 & -1 & 1 & 1 & -1 & -1 & 10 & 0,0388 & 22 & 3 & 300 \\
\hline F1 & -1 & 1 & -1 & 1 & 1 & 10 & 0,0388 & 17 & 6 & 500 \\
\hline $\mathrm{F} 2$ & -1 & 1 & -1 & 1 & -1 & 10 & 0,0388 & 17 & 6 & 300 \\
\hline F3 & -1 & 1 & -1 & -1 & 1 & 10 & 0,0388 & 17 & 3 & 500 \\
\hline $\mathrm{F} 4$ & -1 & 1 & -1 & -1 & -1 & 10 & 0,0388 & 17 & 3 & 300 \\
\hline G1 & -1 & -1 & 1 & 1 & 1 & 10 & 0,0194 & 22 & 6 & 500 \\
\hline G2 & -1 & -1 & 1 & 1 & -1 & 10 & 0,0194 & 22 & 6 & 300 \\
\hline G3 & -1 & -1 & 1 & -1 & 1 & 10 & 0,0194 & 22 & 3 & 500 \\
\hline G4 & -1 & -1 & 1 & -1 & -1 & 10 & 0,0194 & 22 & 3 & 300 \\
\hline $\mathrm{H} 1$ & -1 & -1 & -1 & 1 & 1 & 10 & 0,0194 & 17 & 6 & 500 \\
\hline $\mathrm{H} 2$ & -1 & -1 & -1 & 1 & -1 & 10 & 0,0194 & 17 & 6 & 300 \\
\hline $\mathrm{H} 3$ & -1 & -1 & -1 & -1 & 1 & 10 & 0,0194 & 17 & 3 & 500 \\
\hline $\mathrm{H} 4$ & -1 & -1 & -1 & -1 & -1 & 10 & 0,0194 & 17 & 3 & 300 \\
\hline
\end{tabular}

As eletrofiações foram realizadas à temperatura e umidade ambientes, utilizando-se uma seringa de vidro de $20 \mathrm{ml}$ e uma agulha metálica com $4 \mathrm{~cm}$ de comprimento e $0,8 \mathrm{~mm}$ de diâmetro interno. $\mathrm{O}$ polo positivo do voltímetro foi conectado à agulha da seringa, enquanto o 
outro polo foi ligado a uma placa de cobre coletora, posicionada à distância desejada da agulha e revestida com uma folha de alumínio.

A vazão de saída da solução da agulha foi mantida e controlada por uma bomba conectada à seringa. Cada composição foi eletrofiada por um período de 90 minutos, sendo esperado que as amostras coletadas apresentem-se como membranas finas sobre a folha de alumínio.

As membranas foram levadas, ainda no papel alumínio, para uma mufla, onde foram calcinadas, etapa esta, onde espera-se haver a eliminação do PVA e formação do ZnO. As membranas formadas foram analisadas por MEV e DRX antes e após a calcinação.

\section{ANÁLISE DE RESULTADOS}

Dos experimentos realizados, notou-se em todas as eletrofiações um expressivo gotejamento verticalmente abaixo da ponta da agulha em maior ou menor grau, entretanto aparentemente tal evento não interferiu na formação das nanofibras. Visualmente notou-se que não houve formação de fibras para as combinações referentes aos grupos B e D de forma que não foi necessária a realização de MEV e DRX.

Notou-se, também, que houve boa formação de fibra para as combinações referentes aos grupos A, C, E e G, destacando-se que nos dois primeiros grupos, a membrana formada apresentava-se mais homogênea, com aspecto regular e com melhor recobrimento da área.

Dessa forma, verificou-se a obtenção de fibras sendo todas as amostras já calcinadas, todavia não é possível tirar-se maiores conclusões, pois ainda há análises de MEV e DRX a serem feitas e estudadas. Até o presente momento, todas as análises de MEV já foram concluídas. Como etapas futuras tem-se a determinação do diâmetro médio, bem como as análises de DRX.

A Figura 1, a seguir, mostra algumas imagens MEV das membranas obtidas. Destaca-se que o diâmetro médio de cada amostra ainda será determinado futuramente pelo software ImageJ 1.47.

Figura 1 - Imagem MEV das membranas: a) grupo A antes da calcinação, b) amostra A3 após calcinação. A ampliação envolvida nas duas imagens é de 5000x.

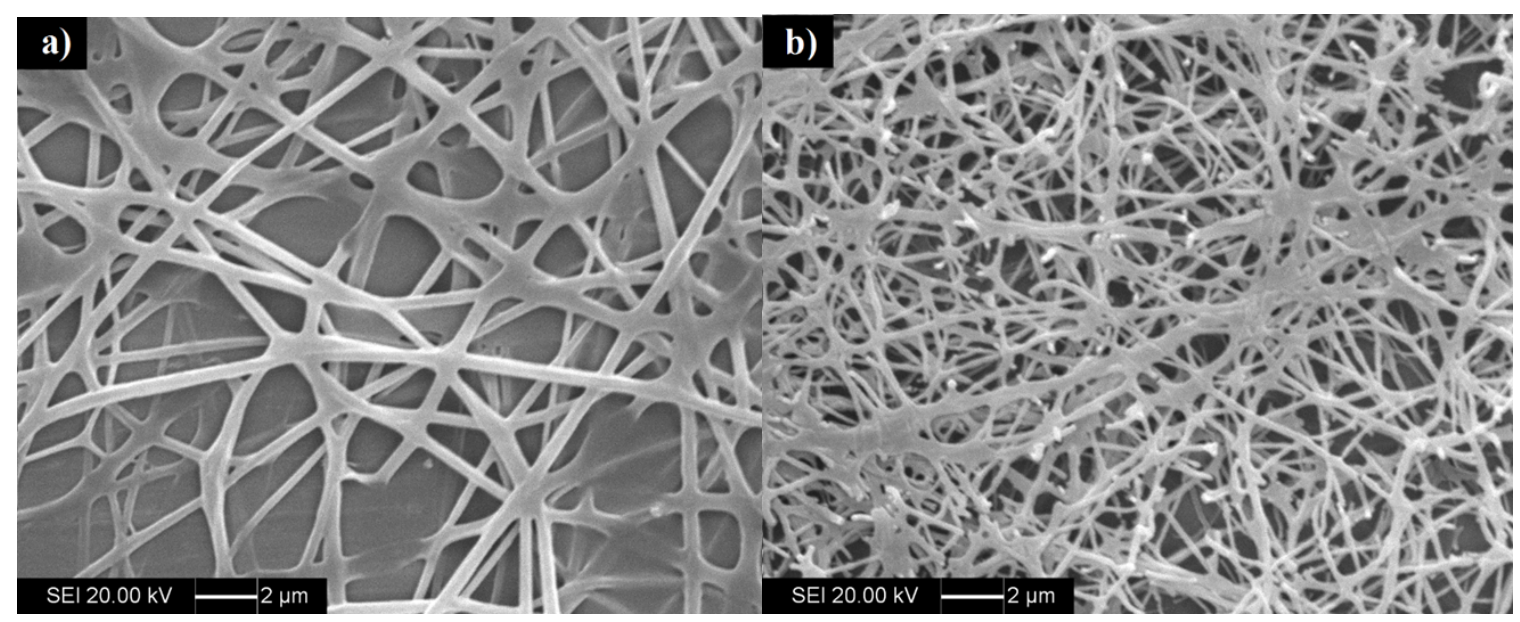


Pela figura 1a observa-se que após a eletrofiação obteve-se a morfologia fibrosa para as composições do grupo A, tal morfologia manteve-se após a calcinação para a amostra A3, evidenciando que os parâmetros de calcinação selecionados foram capazes de manter a estabilidade dimensional da mesma.

\section{CONCLUSÃO}

Com os experimentos realizados até o momento verificou-se a formação de fibras por eletrofiação, entretanto não é possível tirar maiores conclusões, pois ainda há análises de MEV e DRX a serem feitas e estudadas. Atestou-se ainda, como visto na literatura, que distâncias muito grandes entre a agulha e o coletor metálico podem fazer com que a voltagem não seja suficiente para o processo de eletrofiação. Tal fato pôde ser verificado pela não formação de fibra nas combinações $+1-1-1$ (15 cm; $0,0194 \mathrm{~mL} / \mathrm{min}$ e $17 \mathrm{kV})$ e $+1+1-1$ (15 $\mathrm{cm} ; 0,0388 \mathrm{~mL} / \mathrm{min}$ e $17 \mathrm{kV}$ ), ambas associadas a maiores distâncias e menores voltagens.

\section{REFERÊNCIAS BIBLIOGRÁFICAS}

GENSHEIMER, M.; BECKER, M.; BRANDIS-HEEP, A.; WENDORFF, J. H.; THAUER, R. K.; GREINER, A. Novel Biohybrid materials by electrospinning: Nanofibers of Poly(ethylene oxide) and Living Bacteria. Advanced Materials., v. 19, p. 2480-2482, 2007.

LEE, S. Multifunctionality of layered fabric systems based on electrospun polyurethane / zinc oxide nanocomposite fibers. Journal of Applied Polymer Science. v. 114, p. 36523658, 2009.

LI, D.; XIA, Y. Alloying and dealloying processes involved in the preparation of metal nanoshells through a galvanic replacement reaction. Nano Letters., v. 3, p. 555, 2003.

LI, L.; BELLAN, L. M.; CRAIGHEAD, H. G.; FREY, M. W. Formation and properties of nylon-6and nylon-6/montmorillonite composite nanofibers. Polymer., v. 47, p. 62086217, 2006.

MOORER, W. R.; GENET, J. M. Antibacterial activity of gutta-percha cones attributed to the zinc oxide component. Oral Surgery, Oral Medicine, Oral Pathology. v. 53, p. 508$517,1982$.

NISTA, S. V. G. Desenvolvimento e Caracterização de Nanofibras de Acetato de Celulose para Liberação Controlada de Fármacos. Dissertação de Mestrado. Universidade Estadual de Campinas (UNICAMP), 2012.

RAMAKRISHNA, S.; FUJIHARA, K.; TEO, W.; LIM, T.; MA, Z. An Introduction to Electrospinning and Nanofibers. World Scientific., 2005.

RAMASESHAN, R.; SUBRAMANIAN, S.; RAJAN, J.; RAMAKRISHNA, S. Nanostructured Ceramics by Electrospinning. Journal of Applied Physics., v. 102, 2007.

WANNATONG, L.; SIRIVAT, A.; SUPAPHOL, P. Effects of solvents on electrospinning polymeric fibers: Preliminary study on polystyrene. Polymer International., v. 53, p. 1851-1859, 2004.

WEI, S.; ZHOU, M.; DU, W. Improved acetone sensing properties of ZnO hollow nanofibers by single capillary electrospinning. Sensors and Actuators B., v. 160, p. 753-759, 2011. 